OPEN ACCESS

Edited by:

Ryan Arsenault,

University of Delaware, USA

Reviewed by:

Lisa Bielke,

Ohio State University, USA

Michael J. Rothrock,

United States Department of Agriculture, USA

${ }^{*}$ Correspondence: Steven C. Ricke sricke@uark.edu

Specialty section: This article was submitted to Veterinary Infectious Diseases,

a section of the journal Frontiers in Veterinary Science

Received: 22 July 2016 Accepted: 02 September 2016 Published: 16 September 2016

Citation:

Rubinelli P, Roto S, Kim SA, Park SH, Pavlidis HO, Mclntyre D and Ricke SC (2016) Reduction of Salmonella Typhimurium by Fermentation Metabolites of

Diamond V Original XPC in an In Vitro Anaerobic Mixed Chicken Cecal Culture. Front. Vet. Sci. 3:83. doi: 10.3389/fvets.2016.00083

\section{Reduction of Salmonella} Typhimurium by Fermentation Metabolites of Diamond V Original XPC in an In Vitro Anaerobic Mixed Chicken Cecal Culture

\author{
Peter Rubinelli', Stephanie Roto', Sun Ae Kim', Si Hong Park', Hilary O. Pavlidis², \\ Don Mclntyre ${ }^{2}$ and Steven C. Ricke ${ }^{1 *}$ \\ ${ }^{1}$ Department of Food Science, Center for Food Safety, University of Arkansas, Fayetteville, AR, USA, ${ }^{2}$ Diamond V, \\ Cedar Rapids, IA, USA
}

Fermentation metabolites of Diamond $\mathrm{V}$ Original XPC ${ }^{\mathrm{TM}}$ (XPC), a biological product derived from yeast fermentation, were evaluated for their ability to reduce the Salmonella Typhimurium population using an in vitro mixed anaerobic culture system containing cecal microbiota to simulate chicken hindgut conditions. Four different samples were prepared: anaerobic mixed culture containing (1) feed only, (2) cecal only (ceca were harvested from 42 days old broiler chickens), (3) feed and cecal contents, and (4) feed, cecal contents, and 1\% XPC. Two experimental conditions were investigated: Group 1, in which the cecal content was added at the same time as a S. Typhimurium marker strain and Group 2, in which the cecal content was preincubated for $24 \mathrm{~h}$ prior to the inoculation with the $S$. Typhimurium marker strain. The mixed cultures were incubated anaerobically at $37^{\circ} \mathrm{C}$, and the $\mathrm{S}$. Typhimurium marker strain was enumerated at 0,24 , and $48 \mathrm{~h}$. Analysis of short chain fatty acids was also conducted for $24 \mathrm{~h}$. In the Group 1 experiment, adding XPC did not exhibit significant reduction of $S$. Typhimurium. However, the presence of XPC resulted in rapid reduction of $S$. Typhimurium in Group 2. S. Typhimurium was reduced from $6.81 \log _{10} \mathrm{CFU} / \mathrm{ml}(0 \mathrm{~h})$ to $3.73 \log _{10} \mathrm{CFU} / \mathrm{ml}$ and $1.19 \log _{10} \mathrm{CFU} / \mathrm{ml}$ after 24 and $48 \mathrm{~h}$, respectively. These levels were also $2.47 \log _{10}$ and $2.72 \log _{10}$ lower than the $S$. Typhimurium level recovered from the control culture with feed and cecal contents, but without XPC. Based on these results, it appears that the ability of XPC to reduce $S$. Typhimurium requires the presence of the cecal microbiota. Short chain fatty acid analysis indicated that acetate and butyrate concentrations of cultures containing XPC were twofold greater than the control cultures by $24 \mathrm{~h}$ of anaerobic growth. Results from the present study suggest that dietary inclusion of XPC may influence cecal microbiota fermentation and has the potential to reduce Salmonella in the cecum. Implications of these findings suggest that XPC may decrease preharvest levels of Salmonella in broilers and layers.

Keywords: Salmonella Typhimurium, Diamond V Original XPC, mixed anaerobic culture, in vitro, reduction, short chain fatty acids 


\section{INTRODUCTION}

Food-borne disease continues to be one of the primary public health concerns throughout the world. Infections by Salmonella are one of the leading causes of food-borne gastroenteritis to systemic infections in humans. Annually, it is estimated that over one million Americans contract Salmonella (1), and yearly costs for Salmonella control efforts are estimated to be up to $\$ 14.6$ billion $(2,3)$. Salmonellosis usually occurs by consumption of foods or water contaminated with Salmonella, and common sources are poultry and poultry products (4), thus it is essential to control pathogenic Salmonella in poultry products.

Because the use of antibiotic growth promoters provoke a negative reaction from many consumers due to public health concerns such as the appearance of antibiotic resistance, the food industry has been searching for effective alternatives to replace antibiotics (5-7). Prebiotics can be defined as non-digestible food ingredients that selectively simulate the growth of beneficial bacteria and/or minimize pathogen growth in the colon, and they are occasionally used in the poultry industries to improve poultry health as a replacement of antibiotic growth promoters (8-11).

However, there are several ingredients that do not stringently fit the definition of prebiotics, but nevertheless provide similar and beneficial effects on host health with different modes of action compared to prebiotics. These ingredients are referred as "prebiotic-like compounds" (12). Fermentation metabolites of Diamond V Original XPC ${ }^{\mathrm{TM}}$ (XPC; Diamond V, Cedar Rapids, IA, USA) is a common prebiotic-like compound, which includes post-fermentation growth medium residues, residual yeast cells, and yeast cell wall fragments (mannan-oligosaccharides and $\beta$-glucans) (13). To date, several studies of XPC have focused on its effects on the host including feed uptake, growth performance, reproductive performance, and immunomodulatory functions with different animal model systems (13-17); however, few studies have examined inhibitory/bactericidal effects against pathogenic Salmonella $(18,19)$.

Because the environment of the chicken gut is anaerobic, the in vitro methodology using an anaerobic mixed culture can provide more empirical data since it can mimic the chicken cecal environment effectively while minimizing confounding host variables and is considered cost-effective (20). The gut microbiota ferment non-digestible ingredients to produce various compounds including short chain fatty acids (SCFA), methane, hydrogen, and ammonia (21). Among these, SCFA are potential metabolites that can be inhibitory to pathogens such as Salmonella $(22,23)$. In the present study, the ability of XPC in feed to reduce $S$. Typhimurium was investigated using a mixed anaerobic culture system to mimic conditions within the chicken hindgut. Additionally, the requirement for cecal microbiota on the reduction of $S$. Typhimurium by XPC was established. Finally, SCFA analysis was performed on the anaerobic cultures with or without XPC to further characterize the effect of XPC on cecal fermentation.

\section{MATERIALS AND METHODS}

\section{Preparation of Anaerobic Dilution Solution}

Our in vitro anaerobic mixed culture experiment was based on the method of Donalson et al. The mixed cultures were grown in anaerobic dilution solution (ADS), consisting of $0.45 \mathrm{~g} / 1 \mathrm{~K}_{2} \mathrm{HPO}_{4}$, $0.45 \mathrm{~g} / \mathrm{l} \mathrm{KH}_{2} \mathrm{PO}_{4}, 0.45 \mathrm{~g} / \mathrm{l}\left(\mathrm{NH}_{4}\right)_{2} \mathrm{SO}_{4}, 0.9 \mathrm{~g} / \mathrm{l} \mathrm{NaCl}, 0.1875 \mathrm{~g} / \mathrm{l}$ $\mathrm{MgSO}_{4}-7 \mathrm{H}_{2} \mathrm{O}, 0.12 \mathrm{~g} / \mathrm{CaCl}_{2}-2 \mathrm{H}_{2} \mathrm{O}, 1 \mathrm{ml} / 1$ 0.1\% resazurin, $0.05 \%$ cysteine- $\mathrm{HCl}$, and $0.4 \% \mathrm{CO}_{2}$-saturated sodium carbonate, with the sodium carbonate added last as described previously (24-29). ADS was sparged with an anaerobic gas mixture (90\% nitrogen $/ 5 \%$ carbon dioxide $/ 5 \%$ hydrogen) for $30 \mathrm{~min}$ in an anaerobic chamber using an aquarium air pump and airstone prior to autoclaving. Autoclaved ADS was cooled to room temperature and allowed to equilibrate overnight in an anaerobic chamber (Coy Laboratories, Grass Lake, MI, USA) with the same atmosphere described above to remove all traces of oxygen.

\section{Bacterial Culture}

Salmonella Typhimurium marker strain ST97, a nalidixic acidresistant $\left(\mathrm{NA}^{\mathrm{R}}\right)$ isolate (gift of Dr. Billy Hargis, Poultry Health Laboratory, University of Arkansas) was used in the present study. This isolate was grown in sterile glass culture tubes with agitation for $16 \mathrm{~h}$ in Luria-Bertani (LB) medium containing $20 \mu \mathrm{g} /$ $\mathrm{ml}$ nalidixic acid, $37^{\circ} \mathrm{C}$ at $250 \mathrm{rpm}$. The bacterial suspension was washed three times in phosphate-buffered saline (PBS).

\section{Cecal Sample Preparation}

Ceca from three different $\mathrm{CO}_{2}$-euthanized 42-day-old $\mathrm{Cobb}$ male broiler chickens (Cobb-Vantress, Siloam Springs, AR, USA) were collected separately using alcohol-dipped, flame-sterilized tools. A University of Arkansas Institutional Animal Care and Use Committee (IACUC)-approved protocol was used to ensure humane treatment of the chickens (IACUC \# 15052). Ceca were placed in sterile sample bags (VWR, Radnor, PA, USA). The bags were then placed in a portable anaerobic box (Mitsubishi Gas Chemical Co., Japan) containing oxygen-scrubbing sachets. Immediately after harvest, ceca were transferred to an anaerobic chamber (Coy Laboratory Products, Grass Lake, MI, USA). Two palladium catalyst scrubbers running continuously maintained an anaerobic environment inside the chamber.

\section{Anaerobic In Vitro Mixed Cultures}

A portion of the cecal contents from three individual chickens were each removed aseptically within the chamber, weighed, and diluted 1:3000 by addition of $0.1 \mathrm{~g}$ of cecal content to $300 \mathrm{ml}$ ADS for each chicken. A total of $20 \mathrm{ml}$ of this diluted cecal content was transferred to each serum bottle with or without ground chicken feed (40 mesh) and XPC as indicated below. An additional culture received sterile ADS, but no cecal content. An initial inoculum of approximately $1 \times 10^{7} \mathrm{CFU} / \mathrm{ml}$ of $S$. Typhimurium was added to each $20 \mathrm{ml}$ culture. Cultures were stoppered with airtight rubber stoppers and aluminum crimps, removed from the anaerobic chamber, and incubated at $37^{\circ} \mathrm{C}$ with $150 \mathrm{rpm}$ shaking for $48 \mathrm{~h}$.

Two different experimental designs were employed, referred to as Group 1 (unadapted) and Group 2 (adapted), respectively. The experimental designs are illustrated in Figure 1. In Group 1, the Salmonella $\mathrm{NA}^{\mathrm{R}}$ marker strain was added at the beginning of the culture incubation along with cecal bacteria, and/or chicken feed, and/or XPC. In Group 2, S. Typhimurium was added after a $24 \mathrm{~h}$ preincubation of the cecal bacteria with the chicken feed and/or XPC. Three control cultures were run in parallel as indicated in Figure 1. 

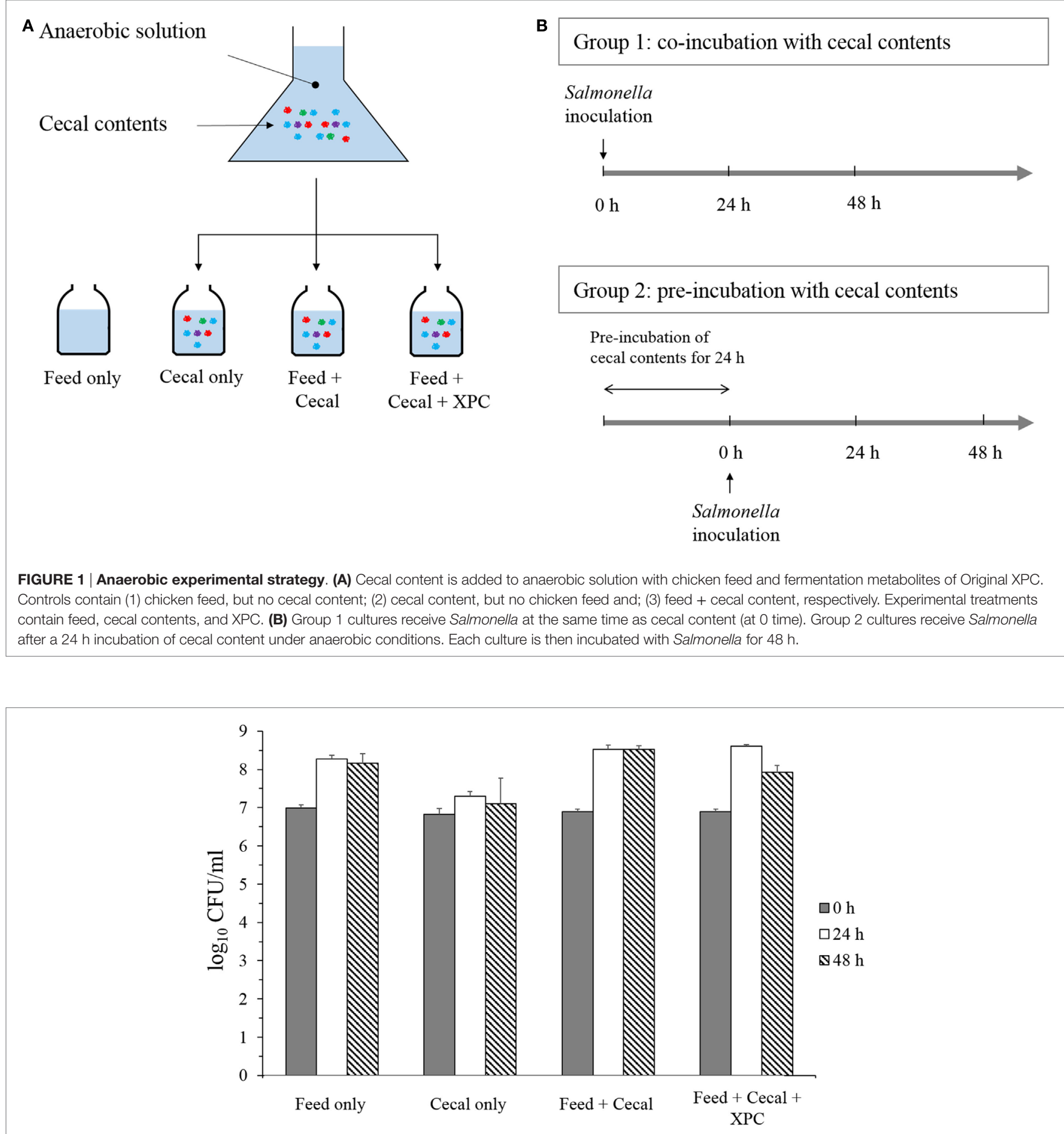

FIGURE 2 | Salmonella Typhimurium survival in unadapted anaerobic cultures (Group 1) with and without fermentation metabolites of Original XPC Bars and brackets represent the mean and SE of three biological replicates.

\section{Salmonella Enumeration}

At 0,24 , and $48 \mathrm{~h}$, an aliquot of each culture was removed, diluted, and spread on Brilliant Green Agar medium (BG, BD Biosciences, Franklin Lakes, NJ, USA) supplemented with $20 \mathrm{ug} /$ $\mathrm{ml}$ nalidixic acid for quantitation of colony forming units (CFU) of marker strain $S$. Typhimurium per milliter of culture. The diluted cecal contents were also tested for $\mathrm{NA}^{\mathrm{R}}$ bacteria prior to addition of marker strain $S$. Typhimurium by inoculation into tetrathionate (TT) enrichment broth (BD Biosciences, Franklin Lakes, NJ, USA), and none were detected. If no Salmonella were 
detected at a particular time point in undiluted culture, that culture was inoculated into TT enrichment broth to confirm that no $S$. Typhimurium survived.

\section{Short Chain Fatty Acid Analysis}

Anaerobic culture supernatants were stored at $-20^{\circ} \mathrm{C}$ until they could be analyzed by gas chromatography. A $1 \mathrm{ml}$ portion of culture supernatant was centrifuged at $14,000 \times g$ to remove solids. An aliquot of the supernatant $(450 \mu \mathrm{l})$ was then mixed with $50 \mu \mathrm{l}$ of GC reagent (50 mM 4-methyl-valeric acid, 5\% meta-phosphoric acid, $1.6 \mathrm{mg} / \mathrm{ml}$ copper sulfate). This mixture was allowed to incubate at $25^{\circ} \mathrm{C}$ for $10 \mathrm{~min}$ and subsequently centrifuged at $14,000 \times g$. The supernatant was transferred to a fresh tube and $1 \mu \mathrm{l}$ was loaded into a Shimadzu 2010 gas chromatograph (Kyoto, Japan) fitted with a $30 \mathrm{~m} \times 0.25 \mathrm{~mm}$ BP21 glass capillary column with $0.25 \mathrm{~mm}$ film thickness (SGE, Austin, TX, USA) operated at $100 \mathrm{kPa} \mathrm{He}$ carrier gas pressure, with $170 \mathrm{kPa} \mathrm{H}_{2}, \mathrm{Ar}$, and air pressure, at $100^{\circ} \mathrm{C}$ for $3 \mathrm{~min}$, followed by a temperature gradient of $4^{\circ} \mathrm{C} / \mathrm{min}$ to $120^{\circ} \mathrm{C}$, holding at $120^{\circ} \mathrm{C}$ for $1 \mathrm{~min}$, followed by a further gradient of $3^{\circ} \mathrm{C} / \mathrm{min}$ to $150^{\circ} \mathrm{C}$. The SPL was maintained at $220^{\circ} \mathrm{C}$ with split ratio $=30$. FID was maintained at $230^{\circ} \mathrm{C}$. Carrier gas flow rate was set to $30 \mathrm{ml} / \mathrm{min}$. A 1:100 mixture of acetic, propionic, and butyric acids was serially diluted, mixed with GC reagent, and used as standards. Peak areas were normalized for loading differences using the valeric acid internal control from the GC reagent.

\section{Statistical Analysis}

Means were determined to be significantly different if $P<0.05$ by two-tailed paired Student $t$-test using Microsoft Excel.

\section{RESULTS AND DISCUSSION}

The main objective of this study was to investigate the inhibitory effect of XPC on S. Typhimurium when combined in vitro with cecal microbiota. The ceca are the main site where pathogens including Salmonella colonize (30). Since poultry have a relatively slow digestion transit time, poultry ceca have a large number of bacteria, and the majority of these are strictly anaerobic $(27,31$, 32). Cecal bacteria in poultry become more diverse as the host matures, and they can maximize metabolic fermentation in an anaerobic environment (12). Cecal contents used in this study were obtained from mature chickens (42-day-old chickens), thus it should serve as a source of a fairly diverse microbiota containing a wide range of anaerobic bacteria. Also, using an anaerobic mixed culture in this study could help to understand the actual fate of Salmonella in ceca by various feeding conditions.

Two conditions were investigated in the present study: Group 1 (unadapted), in which the cecal microbiota was added at the same time as the $S$. Typhimurium and Group 2 (adapted), in which the cecal microbiota was allowed to metabolize anaerobically for $24 \mathrm{~h}$ prior to the inoculation of $S$. Typhimurium (see Figure 1 for design). Results on $S$. Typhimurium reduction by XPC were different between groups. In the unadapted condition (Group $1)$, the population of $S$. Typhimurium was slightly increased or maintained during $48 \mathrm{~h}$ incubation in all controls (feed only, cecal only, and feed + cecal) and treatment (feed + ceca + XPC); the population after incubation was not significantly different from the initial population (Figure 2). In the feed + cecal sample, $S$. Typhimurium populations were increased from $6.89 \log _{10} \mathrm{CFU} /$ $\mathrm{ml}$ to 8.52 and $8.53 \log _{10} \mathrm{CFU} / \mathrm{ml}$ after 24 and $48 \mathrm{~h}$, respectively

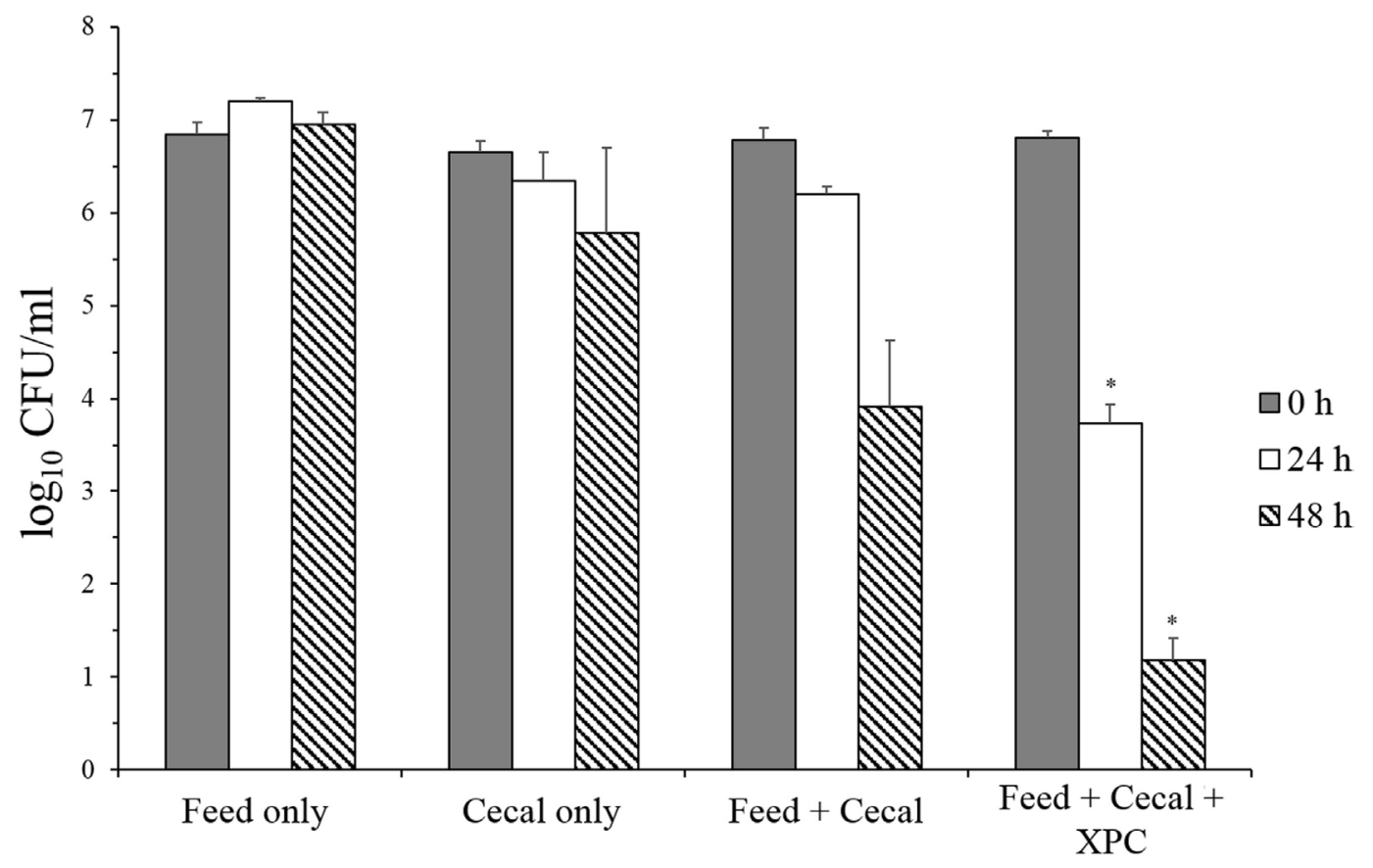

FIGURE 3 | Salmonella Typhimurium survival in adapted anaerobic cultures (Group 2) with and without fermentation metabolites of Original XPC Bars and brackets represent the mean and SE of three biological replicates. Asterisks indicate significant difference $(P<0.05)$ from the "feed + cecal" control. 
$(P<0.05)$. When XPC was added to the feed + cecal sample, $S$. Typhimurium populations were increased from $6.89 \log _{10} \mathrm{CFU} /$ $\mathrm{ml}$ to 8.60 and $7.92 \log _{10} \mathrm{CFU} / \mathrm{ml}$ after 24 and $48 \mathrm{~h}(P<0.05)$, respectively, indicating that XPC had little or no effect on Salmonella survival when $S$. Typhimurium was added at the same time as cecal contents.

In contrast, XPC-containing cultures exhibited a significant reduction in Salmonella survival under adapted conditions (Group 2) (Figure 3). There was no reduction in S. Typhimurium in the feed-only control sample, and only a $0.87 \log _{10}$ reduction of populations of $S$. Typhimurium was achieved after $48 \mathrm{~h}$ incubation in the cecal-only control sample. In addition, the $S$. Typhimurium population was decreased in both the feed + cecal control and the feed + cecal + XPC treatments. However, the presence of XPC

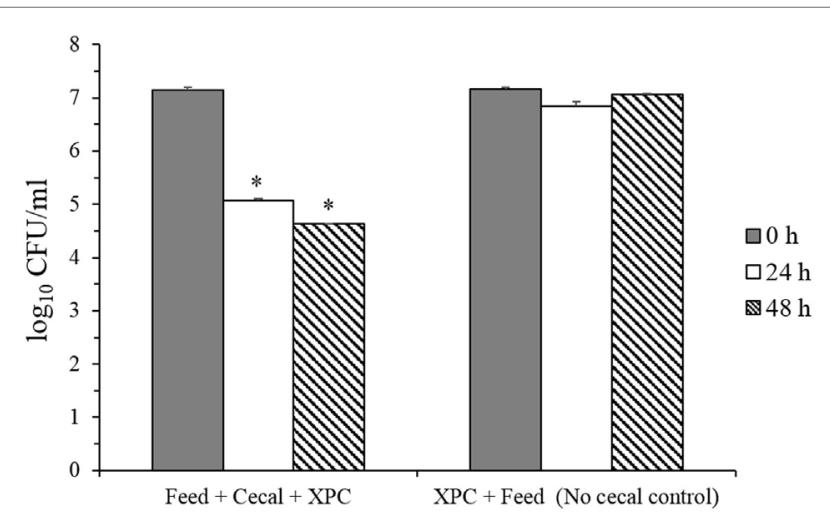

FIGURE 4 | Salmonella Typhimurium survival in feed + cecal + fermentation metabolites of Original XPC and in control anaerobic cultures lacking cecal contents. Asterisks indicate significant difference $(P<0.005)$ from the corresponding control. resulted in a greater reduction of $S$. Typhimurium compared with the feed + cecal control. When $S$. Typhimurium was inoculated to the feed + cecal control, a $2.87 \log _{10}$ reduction in the bacterial population was observed after $48 \mathrm{~h}$. With XPC, the $\log _{10}$ reductions achieved after 24 and $48 \mathrm{~h}$ incubation were 3.08 and 5.62 $\log _{10}$ reduction, respectively. These levels are $2.47 \log _{10}(24 \mathrm{~h})$ and $2.72 \log _{10}(48 \mathrm{~h})$ lower than the Salmonella level recovered from the feed + cecal control.

These results suggested that adaptation of the cecal microbiome in the in vitro mixture to XPC prior to inoculation of $S$. Typhimurium appears to generate a more inhibitory environment for Salmonella than XPC unadapted cecal cultures. To evaluate the role of the microbiota on reduction of $S$. Typhimurium, survival of $S$. Typhimurium in "Feed + cecal + XPC" and "Feed + XPC without cecal contents" were compared (Figure 4). When $S$. Typhimurium was exposed to XPC in the absence of broiler cecal content, no reduction in $S$. Typhimurium was observed, suggesting that XPC acts in concert with cecal microbiota to inhibit $S$. Typhimurium (Figure 4). This is a further indication that cecal microbiota are essential to the reduction of Salmonella by XPC. These results are in accordance with a previous study reporting higher inhibitory activities of fructooligosaccharide in samples preincubated with cecal microbiota prior to inoculation of bacteria (25). Furthermore, the results from both in vitro studies suggest that dietary inclusion of XPC may influence cecal microbiota fermentation and has the potential to reduce Salmonella colonization in the cecum.

The SCFA analysis of supernatants from the mixed cultures indicated that acetate and butyrate concentrations of cultures containing XPC + cecum and XPC + feces were twofold greater than the control cultures after $24 \mathrm{~h}$ of anaerobic growth (Figure 5). This suggests one or more microorganisms have potentially increased acetate and/or butyrate production as a result of being exposed

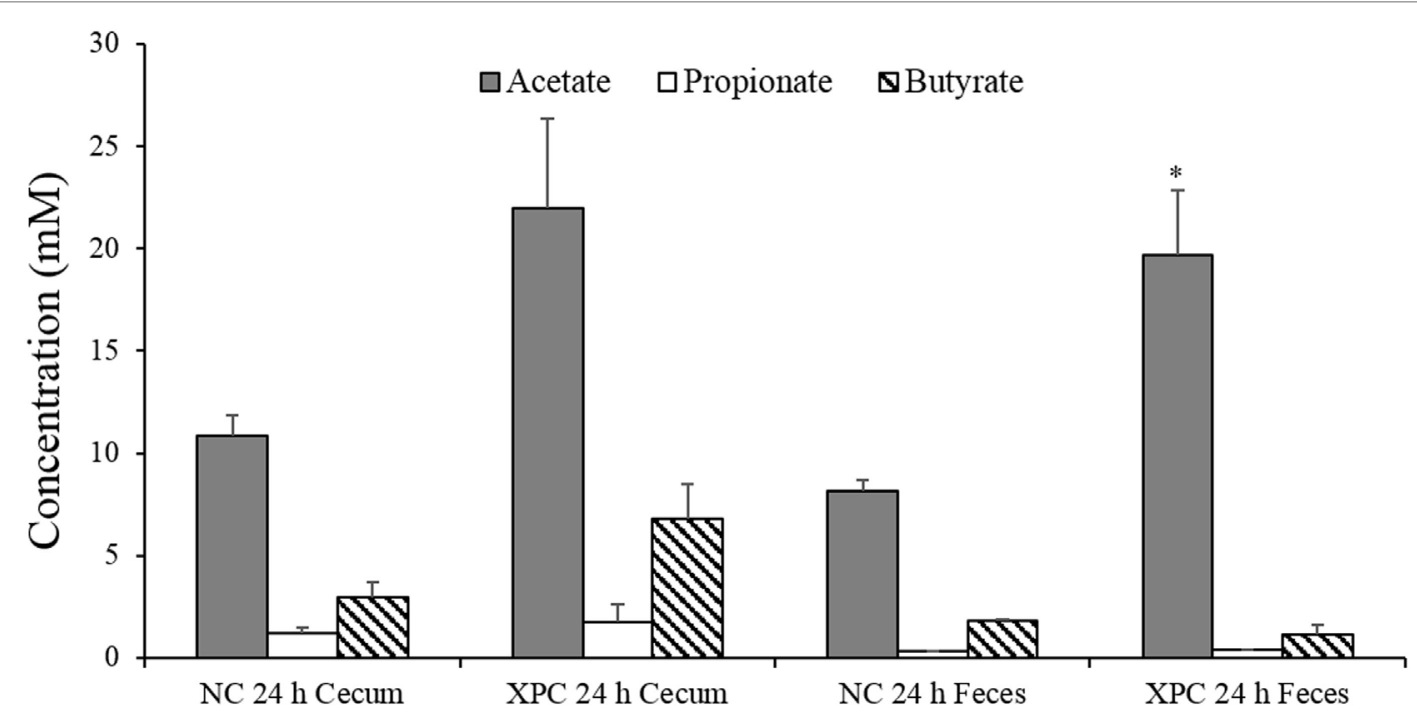

FIGURE 5 | Short chain fatty acid analysis of $\mathbf{2 4} \mathrm{h}$ anaerobic cultures containing 6-week-old broiler cecal contents or feces with and without $\mathbf{1 \%}$ fermentation metabolites of Original XPC. Bars and brackets represent the mean and SE of three biological replicates (chickens). Asterisk indicates significant difference $(P<0.05)$ from corresponding negative control (NC). 
to components of XPC. This additional acetate and butyrate may be contributing to the inhibition of Salmonella due to the direct toxic effect of intracellular anion accumulation when these acids dissociate in the cytosol of sensitive bacteria such as Salmonella $(22,23,33)$. Interestingly, butyrate has been found to inhibit Salmonella invasion of host cells by downregulating Salmonella pathogenicity island 1 (SPI-1) gene expression $(22,34)$. Along these lines, Feye et al. has shown that XPC fed to broilers reduces the virulence regulatory gene hilA in the intestine (19).

In conclusion, XPC can effectively reduce $S$. Typhimurium survival (5.62 $\log _{10}$ reduction) in an in vitro anaerobic mixed cecal culture, and XPC and cecal microbiota are both required for the reduction of $S$. Typhimurium survival. Incubation of cecal microbiota with XPC increased SCFA levels (particularly acetate) in anaerobic cultures. The use of XPC as a prebiotic-like compound has a number of advantages for use in poultry: (1) there are no concerns over usage of antibiotics or growth promoters or the appearance of antibiotic-resistant bacteria, (2) the use of XPC is acceptable to the both industries and consumer since it is a naturally derived yeast product (also an environmentally friendly product), (3) its use by the poultry industry is also feasible because XPC was classified as generally recognized as safe (GRAS) by US FDA (13). To the best of our knowledge, this is the first study to examine the inhibitory effects of XPC in feed with an anaerobic mixed cecal inocula culture to mimic the chicken cecal environment. The implication of these findings is that XPC may decrease preharvest levels of Salmonella in the ceca of broilers

\section{REFERENCES}

1. Scallan E, Hoekstra RM, Angulo FJ, Tauxe RV, Widdowson M-A, Roy SL, et al. Foodborne illness acquired in the United States - major pathogens. Emerg Infect Dis (2011) 17:7-15. doi:10.3201/eid1701.P11101

2. Scharff RL. Health-Related Costs from Foodborne Illness in the United States. Produce Safety Project. Washington, DC: Georgetown University (2010).

3. Heithoff DM, Shimp WR, House JK, Xie Y, Weimer BC, Sinsheimer RL, et al. Intraspecies variation in the emergence of hyperinfectious bacterial strains in nature. PLoS Pathog (2012) 8:e1002647. doi:10.1371/journal.ppat. 1002647

4. Hedican E, Smith K, Jawahir S, Scheftel J, Kruger K, Birk R, et al. Multistate outbreaks of Salmonella infections associated with live poultry-United States, 2007. Morb Mortal Wkly Rep (2009) 58:25-9.

5. Jones FT, Ricke SC. Observations on the history of the development of antimicrobials and their use in poultry feeds. Poult Sci (2003) 82:613-7. doi:10.1093/ ps/82.4.613

6. Park SH, Lee SI, Ricke SC. Microbial populations in naked neck chicken ceca raised on pasture flock fed with commercial yeast cell wall prebiotics via an Illumina MiSeq platform. PLoS One (2016) 11:e0151944. doi:10.1371/journal. pone. 0151944

7. Ricke SC, Calo JR. Antibiotic resistance in pathogenic Salmonella. In: Chen C-Y, Yan X, Jackson C, editors. Antimicrobial Resistance and Food Safety: Methods and Techniques. Amsterdam, Netherlands: Academic Press/Elsevier Science (2015). p. 37-53.

8. Patterson J, Burkholder K. Application of prebiotics and probiotics in poultry production. Poult Sci (2003) 82:627-31. doi:10.1093/ps/82.4.627

9. Gibson G, Roberfroid M. Dietary modulation of the human colonic microbiota: introducing the concept of prebiotics. J Nutr (1995) 125:1401-12.

10. Roberfroid M. Prebiotics: the concept revisited. J Nutr (2007) 137:830S-7S.

11. Ricke SC. Potential of fructooligosaccharide prebiotics in alternative and nonconventional poultry production systems. Poult Sci (2015) 94:1411-8. doi:10.3382/ps/pev049 and layers, thus it could be a suitable alternative to antibiotics currently used in poultry industries.

\section{AUTHOR CONTRIBUTIONS}

PR and SR performed experiments, drafted the manuscript, collected test data, and analyzed the data. SK, HP, and DM revised the manuscript. PR, SP, and SR designed the study and revised the manuscript.

\section{ACKNOWLEDGMENTS}

We would like to thank the University of Arkansas, Fayetteville, FDSC program for supporting a graduate student assistantship to SR. We thank the Michael G. Johnson Endowed Scholarship for Excellence in Food Microbiology Research, Teaching, and Peer Mentoring for providing financial assistance to SR. We would like to thank Diamond V Mills (Cedar Rapids, IA, USA) for support of this study. Author SK is supported by Basic Science Research Program through the National Research Foundation of Korea (NRF) funded by the Ministry of Education (NRF-2015R1A6A3A03016811).

\section{FUNDING}

Funding is provided by Diamond V (Cedar Rapids, IA, USA).

12. Roto SM, Rubinelli PM, Ricke SC. An introduction to the avian gut microbiota and the effects of yeast-based prebiotic-type compounds as potential feed additives. Front Vet Sci (2015) 2:28. doi:10.3389/fvets.2015.00028

13. Shen YB, Carroll JA, Yoon I, Mateo RD, Kim SW. Effects of supplementing Saccharomyces cerevisiae fermentation product in sow diets on performance of sows and nursing piglets. JAnim Sci (2011) 89:2462-71. doi:10.2527/ jas.2010-3642

14. Osweiler G, Jagannatha S, Trampel D, Imerman P, Ensley S, Yoon I, et al. Evaluation of XPC and prototypes on aflatoxin-challenged broilers. Poult Sci (2010) 89:1887-93. doi:10.3382/ps.2010-00773

15. Gao J, Zhang H, Yu S, Wu S, Yoon I, Quigley J, et al. Effects of yeast culture in broiler diets on performance and immunomodulatory functions. Poult Sci (2008) 87:1377-84. doi:10.3382/ps.2007-00418

16. Kim SW, Brandherm M, Newton B, Cook DR, Yoon I, Fitzner G. Effect of supplementing Saccharomyces cerevisiae fermentation product in sow diets on reproductive performance in a commercial environment. Can J Anim Sci (2010) 90:229-32. doi:10.4141/CJAS09100

17. Kiarie E, Bhandari S, Scott M, Krause D, Nyachoti C. Growth performance and gastrointestinal microbial ecology responses of piglets receiving Saccharomyces cerevisiae fermentation products after an oral challenge with Escherichia coli (K88). J Anim Sci (2011) 89:1062-78. doi:10.2527/jas.2010-3424

18. Smith D, Carlson S, Anderson K, Pavlidis H. Reduction of broiler chicken and turkey Salmonella prevalence, numbers, and virulence by Diamond V original XPC. IAFP's 12th European Symposium on Food Safety. Athens, Greece (2016).

19. Feye KM, Anderson KL, Scott MF, McIntyre DR, Carlson SA. Inhibition of the virulence, antibiotic resistance, intestinal colonization, and fecal shedding of multiple antibiotic-resistant Salmonella Typhimurium in broilers fed Original $\mathrm{XPC}^{\mathrm{TM}}$. Poult Sci (2016). doi:10.3382/ps/pew254

20. PolliJE. Invitro studies are sometimes better than conventional human pharmacokinetic in vivo studies in assessing bioequivalence of immediate-release solid oral dosage forms. AAPS J (2008) 10:289-99. doi:10.1208/s12248-008-9027-6

21. Tsukahara T, Ushida K. Effects of animal or plant protein diets on cecal fermentation in guinea pigs (Cavia porcellus), rats (Rattus norvegicus) and 
chicks (Gallus gallus domesticus). Comp Biochem Physiol Part A Mol Integr Physiol (2000) 127:139-46. doi:10.1016/S1095-6433(00)00244-0

22. Van Immerseel F, Russell J, Flythe M, Gantois I, Timbermont L, Pasmans F, et al. The use of organic acids to combat Salmonella in poultry: a mechanistic explanation of the efficacy. Avian Pathol (2006) 35:182-8. doi:10.1080/03079450600711045

23. Ricke SC. Perspectives on the use of organic acids and short chain fatty acids as antimicrobials. Poult Sci (2003) 82:632-9. doi:10.1093/ps/82.4.632

24. Donalson L, Kim W, Chalova V, Herrera P, McReynolds J, Gotcheva V, et al. In vitro fermentation response of laying hen cecal bacteria to combinations of fructooligosaccharide prebiotics with alfalfa or a layer ration. Poult Sci (2008) 87:1263-75. doi:10.3382/ps.2007-00179

25. Donalson LM, Kim WK, Chalova VI, Herrera P, Woodward CL, McReynolds JL, et al. In vitro anaerobic incubation of Salmonella enterica serotype Typhimurium and laying hen cecal bacteria in poultry feed substrates and a fructooligosaccharide prebiotic. Anaerobe (2007) 13:208-14. doi:10.1016/j. anaerobe.2007.05.001

26. Dunkley KD, Dunkley CS, Njongmeta NL, Callaway TR, Hume ME, Kubena LF, et al. Comparison of in vitro fermentation and molecular microbial profiles of high-fiber feed substrates incubated with chicken cecal inocula. Poult Sci (2007) 86:801-10. doi:10.1093/ps/86.5.801

27. Fan YY, Ricke SC, Scanlan CM, Nisbet DJ, Vargas-Moskola AA, Corrier DE, et al. Use of differential rumen fluid-based carbohydrate agar media for culturing lactose-selected cecal bacteria from chickens. J Food Prot (1995) 58:361-7.

28. Saengkerdsub S, Kim W-K, Anderson RC, Nisbet DJ, Ricke SC. Effects of nitrocompounds and feedstuffs on in vitro methane production in chicken cecal contents and rumen fluid. Anaerobe (2006) 12:85-92. doi:10.1016/ j.anaerobe.2005.11.006

29. Shermer CL, Maciorowski KG, Bailey CA, Byers FM, Ricke SC. Caecal metabolites and microbial populations in chickens consuming diets containing a mined humate compound. J Sci Food Agric (1998) 77:479-86. doi:10.1002/ (SICI) 1097-0010(199808)77:4<479::AID-JSFA607>3.0.CO;2-L

30. Hudault S, Bewa H, Bridonneau C, Raibaud P. Efficiency of various bacterial suspensions derived from cecal floras of conventional chickens in reducing the population level of Salmonella typhimurium in gnotobiotic mice and chicken intestines. Can J Microbiol (1985) 31:832-8. doi:10.1139/m85-155

31. Salanitro J, Blake I, Muirhead P. Studies on the cecal microflora of commercial broiler chickens. Appl Microbiol (1974) 28:439-47.

32. Ricke SC, Pillai SD. Conventional and molecular methods for understanding probiotic bacteria functionality in gastrointestinal tracts. Crit Rev Microbiol (1999) 25:19-38. doi:10.1080/10408419991299176

33. Russell JB, Diez-Gonzalez F. The effects of fermentation acids on bacterial growth. Adv Microb Physiol (1997) 39:205-34. doi:10.1016/ S0065-2911(08)60017-X

34. Gantois I, Ducatelle R, Pasmans F, Haesebrouck F, Hautefort I, Thompson A, et al. Butyrate specifically down-regulates Salmonella pathogenicity island 1 gene expression. Appl Environ Microbiol (2006) 72:946-9. doi:10.1128/ AEM.72.1.946-949.2006

Conflict of Interest Statement: The authors declare that the research was conducted in the absence of any commercial or financial relationships that could be construed as a potential conflict of interest.

Copyright $\odot 2016$ Rubinelli, Roto, Kim, Park, Pavlidis, McIntyre and Ricke. This is an open-access article distributed under the terms of the Creative Commons Attribution License (CC BY). The use, distribution or reproduction in other forums is permitted, provided the original author(s) or licensor are credited and that the original publication in this journal is cited, in accordance with accepted academic practice. No use, distribution or reproduction is permitted which does not comply with these terms. 